\title{
Magnetohydrodynamic scaling: From astrophysics to the laboratory*
}

\author{
D. D. Ryutov, ${ }^{\dagger}$ B. A. Remington, and H. F. Robey \\ Lawrence Livermore National Laboratory, Livermore, California 94551
}

R. P. Drake

University of Michigan, Ann Arbor, Michigan 48105

(Received 24 October 2000; accepted 4 December 2000)

\begin{abstract}
During the last few years, considerable progress has been made in simulating astrophysical phenomena in laboratory experiments with high-power lasers. Astrophysical phenomena that have drawn particular interest include supernovae explosions; young supernova remnants; galactic jets; the formation of fine structures in late supernovae remnants by instabilities; and the ablation-driven evolution of molecular clouds. A question may arise as to what extent the laser experiments, which deal with targets of a spatial scale of $\sim 100 \mu \mathrm{m}$ and occur at a time scale of a few nanoseconds, can reproduce phenomena occurring at spatial scales of a million or more kilometers and time scales from hours to many years. Quite remarkably, in a number of cases there exists a broad hydrodynamic similarity (sometimes called the "Euler similarity") that allows a direct scaling of laboratory results to astrophysical phenomena. A discussion is presented of the details of the Euler similarity related to the presence of shocks and to a special case of a strong drive. Constraints stemming from the possible development of small-scale turbulence are analyzed. The case of a gas with a spatially varying polytropic index is discussed. A possibility of scaled simulations of ablation front dynamics is one more topic covered in this paper. It is shown that, with some additional constraints, a simple similarity exists. (C) 2001 American Institute of Physics.
\end{abstract}

[DOI: $10.1063 / 1.1344562$ ]

\section{INTRODUCTION}

The general idea of experimental simulation of astrophysical phenomena is very attractive for the obvious reason: at the laboratory we can change at our will characteristic parameters of the system and initial conditions and follow a time history of the event through a significant time segment. Thereby, we can get deeper insights into astrophysical problems, help to make a judicial selection between various theoretical models, and even develop suggestions of the optimum observation strategy. Recent years have witnessed a number of experiments in that area (see Refs. 1 and 2 for the survey), ranging from galactic jets and supernova (SN) dynamics, through measurements of opacities, to determining equations of state (EOS) required for the theory of the giant planets.

In this paper we shall limit ourselves to those phenomena that can be reasonably well described in the framework of hydrodynamical equations. Laboratory studies of the hydrodynamics of astrophysical objects have been carried out mostly with intense lasers; ${ }^{3-6}$ some were carried out with fast $\mathrm{Z}$ pinches. ${ }^{7}$ In what follows, we will concentrate on laser experiments.

Laser experiments are typically performed with experimental objects of the size of a fraction of a millimeter, and typically last from a few nanoseconds to a few tens of nanoseconds, whereas astrophysical phenomena occur at the spatial and temporal scales that are sometimes 15-25 orders of

*Paper CI1 1, Bull. Am. Phys. Soc. 45, 57 (2000).

${ }^{\dagger}$ Invited speaker. magnitude larger. A natural question then arises whether the laboratory experiments can actually simulate the astrophysical phenomena. In this paper we show that the answer to this question is often positive, provided proper scaling relations have been established between the two systems. (Of course, to do that, one should know basic equations describing both systems.)

Studies of scaling properties and dimensional analysis of physical systems have begun in the XIXth century. ${ }^{8}$ The most familiar achievement of that period was establishing the role of the Reynolds number in the hydrodynamics of viscous fluid. Dimensional analysis and similarity theory reached a mature state by 1910-1920 (see, e.g., Ref. 9, where the so-called $\Pi$ theorem was introduced). Issues of dimensional analysis and hydrodynamic similarities have been later summarized in a number of texts (e.g., Ref. 10).

The similarity analysis is indeed a very powerful tool. First, if the similarity is well established, it allows one to make quick predictions regarding the behavior of the system with parameters different from those of the system with which actual experiments have been done. Second, considerable deviations from a specific group of similarity laws in some areas of the parameter space are indicative of new physical effects (not included in the model for which the similarity was developed); hints on the nature of these new effects can be obtained from the character of deviations from the scaling laws.

In plasma physics, first dimensional analyses of basic equations were carried out in the pioneering works by Lacina, ${ }^{11}$ Kadomtsev, ${ }^{12}$ and Connor and Taylor. ${ }^{13}$ In the lat- 
ter paper the scale invariance of various plasma models has been analyzed [including the magnetohydrodynamic (MHD) model described by Eqs. (1), (4) below], and scaling laws for the plasma confinement time in fusion devices has been established for various models, including the MHD model.

In this paper, we shall concentrate on those of the astrophysical phenomena that can be adequately described within a hydrodynamic approximation. They include various stages of the SN explosion, radiative blast waves, extragalactic jets, etc. The first detailed discussion of hydrodynamical scaling between astrophysics and the laboratory was presented in Refs. 14 and 15, and we will base part of our analysis on these papers.

In addition to the scaled laboratory experiment, another powerful tool for analyzing hydrodynamical phenomena in astrophysics is numerical simulation. Significant progress has been made in this area during the last decade. Still, the role of a real experiment remains critically important, especially in the areas where complex geometries are involved, or where small-scale motions develop. Real experiments are also irreplaceable in providing new insights into subtle physics issues and in stirring the creative imagination of scientists.

One should of course remember that there are numerous astrophysical phenomena where the hydrodynamic description does not work, in particular, the ones involving highenergy particles. We will not touch upon the corresponding problems. The most recent paper discussing possible scaling of these phenomena to the laboratory is that by Drake. ${ }^{16}$

The plan of the paper is as follows: In Sec. II, similarity properties of the equations of ideal hydrodynamics are discussed in the context of the initial value problem. A similarity that covers a very broad class of motions including shock wave formation and the transition to hydrodynamic turbulence has been described (the Euler similarity). In Sec. III volumetric radiative losses are included into the picture, and additional constraints are introduced. In Sec. IV applicability conditions of the ideal hydrodynamics are discussed and the potential importance of dissipative processes at small spatial scales is elucidated. In Sec. V, we consider similarity in the hydrodynamics of ablation front, where the surface absorption of incident radiation becomes an important factor. Finally, Sec. VI contains a summary.

\section{SIMILARITY PROPERTIES OF IDEAL HYDRODYNAMICS EQUATIONS}

\section{A. Euler similarity}

We start from the Euler equations, with magnetohydrodynamics effects included:

$$
\begin{aligned}
& \frac{\partial \rho}{\partial t}+\nabla \cdot \rho \mathbf{v}=0, \\
& \rho\left(\frac{\partial \mathbf{v}}{\partial t}+\mathbf{v} \cdot \nabla \mathbf{v}\right)=-\nabla p-\frac{1}{4 \pi} \mathbf{B} \times \nabla \times \mathbf{B}, \\
& \frac{\partial \mathbf{B}}{\partial t}=\nabla \times \mathbf{v} \times \mathbf{B},
\end{aligned}
$$

where $\mathbf{v}, \rho, p$, and $\mathbf{B}$ are the velocity, the density, the pressure, and the magnetic field, respectively. The CGS system of units is used throughout this paper. We have to supplement these equations with the energy equation, which reads as

$$
\frac{\partial \epsilon}{\partial t}+\mathbf{v} \cdot \boldsymbol{\nabla} \epsilon=-(\epsilon+p) \boldsymbol{\nabla} \cdot \mathbf{v}
$$

where $\epsilon$ is the internal energy per unit volume. This equation implies that there are no dissipative processes in the fluid, so that the entropy of any fluid element remains constant [for this reason Eq. (2) is sometimes called "the entropy equation']. We assume that are dealing with a polytropic fluid, i.e., with the fluid where the internal energy is proportional to the pressure,

$$
\epsilon=C p,
$$

where $C$ is some dimensionless constant. Note that this assumption goes beyond the assumption of the ideal gas. In particular, it breaks down if internal degrees of freedom can be excited at higher temperature, or the ionization degree can change. On the other hand, it correctly describes fully ionized medium, as well as the medium where radiation pressure is dominant. For the polytropic gas, the energy equation reduces to

$$
\frac{\partial p}{\partial t}+\mathbf{v} \cdot \boldsymbol{\nabla} p=-\gamma p \boldsymbol{\nabla} \cdot \mathbf{v},
$$

where

$$
\gamma=1+\frac{1}{C}
$$

is a familiar adiabat index. For the fully ionized nonrelativistic gas it is equal to $\frac{5}{3}$; for the gas where the radiation pressure is dominant it is equal to $\frac{4}{3}$.

Consider the initial value problem for the set of Eqs. (1) and (4). Let us present the initial spatial distributions of the density, pressure, velocity, and magnetic field in the following way:

$$
\begin{array}{ll}
\left.\rho\right|_{t=0}=\rho^{*} f\left(\frac{\mathbf{r}}{L^{*}}\right) ; & \left.p\right|_{t=0}=p^{*} g\left(\frac{\mathbf{r}}{L^{*}}\right) ; \\
\left.\mathbf{v}\right|_{t=0}=v^{*} \mathbf{h}\left(\frac{\mathbf{r}}{L^{*}}\right) ; & \left.\mathbf{B}\right|_{t=0}=B^{*} \mathbf{k}\left(\frac{\mathbf{r}}{L^{*}}\right),
\end{array}
$$

where $L^{*}$ is the characteristic spatial scale of the problem, and the other quantities marked by the asterisk denote the value of the corresponding parameter in some characteristic point; the dimensionless functions (vectorial functions) $f, g$, $\mathbf{h}$, and $\mathbf{k}$ are of order unity. They determine the spatial shape of the initial distribution. We note that there are five dimensional parameters determining initial conditions:

$$
L^{*}, \quad \rho^{*}, \quad p^{*}, \quad v^{*}, \quad B^{*} .
$$

Let us then introduce dimensionless variables (which we denote by the overtilde) in the following way:

$$
\tilde{\mathbf{r}}=\frac{\mathbf{r}}{L^{*}} ; \quad \tilde{t}=\frac{t}{L^{*}} \sqrt{\frac{p^{*}}{\rho^{*}}} ; \quad \tilde{\rho}=\frac{\rho}{\rho^{*}} ; \quad \tilde{p}=\frac{p}{p^{*}} ;
$$




$$
\tilde{\mathbf{v}}=\mathbf{v} \sqrt{\frac{\rho^{*}}{p^{*}}} ; \quad \tilde{\mathbf{B}}=\frac{\mathbf{B}}{\sqrt{p^{*}}} .
$$

When one expresses the set of equations (1) and (4) in terms of the dimensionless variables, one finds that this set maintains its form, just all the quantities get replaced by their analogs bearing the overtilde. Initial conditions presented in the dimensionless variables acquire the form

$$
\begin{aligned}
& \left.\tilde{\rho}\right|_{t=0}=f(\tilde{\mathbf{r}}) ;\left.\quad p\right|_{t=0}=g(\tilde{\mathbf{r}}) ; \\
& \left.\tilde{\mathbf{v}}\right|_{\tilde{t}=0}=v^{*} \sqrt{\frac{\rho^{*}}{p^{*}}} \mathbf{h}(\tilde{\mathbf{r}}) ;\left.\quad \widetilde{\mathbf{B}}\right|_{\tilde{t}=0}=\frac{B^{*}}{\sqrt{p^{*}}} \mathbf{k}(\tilde{\mathbf{r}}) .
\end{aligned}
$$

One sees that dimensionless initial conditions for the two systems are identical if the dimensionless functions $f, g, \mathbf{h}$, and $\mathbf{k}$ maintain their form, and two dimensionless parameters, $v^{*} \sqrt{\rho^{*} / p^{*}}$ and $B^{*} / \sqrt{p^{*}}$, remain unchanged,

$$
v^{*} \sqrt{\frac{\rho^{*}}{p^{*}}}=\mathrm{inv} ; \quad \frac{B^{*}}{\sqrt{p^{*}}}=\mathrm{inv}
$$

(here and in the following we use abbreviation "inv" for the word "invariant"). In other words, provided these two parameters are invariant, and the initial states are geometrically similar (i.e., the functions $f, g, \mathbf{h}$, and $\mathbf{k}$ are the same), one would have the same dimensionless equations and the same dimensionless initial conditions for any two hydrodynamical systems, meaning that the systems will evolve identically, up to the scale transformations.

Normally, the similarity arguments are used to ensure that some parameter (of interest in a particular problem) can be scaled between two systems. This can be, e.g., the hydrodynamic resistance to the body moving in a fluid, or the energy confinement time in a plasma device (as in Ref. 13). We are demanding much more, that the whole dynamical evolution of two systems with properly scaled initial conditions be similar.

There are only two constraints on five parameters (7) determining evolution of the system. For the second system, one can choose arbitrarily three parameters, say, $L^{*}, p^{*}$, and $\rho^{*}$ and, by choosing the magnitude of the characteristic velocity and the magnetic field so as to maintain conditions (10), obtain a system that behaves similarly to the first one, up to the change in the scales. For example, if in the first system the density was some function of coordinate and time, $\rho_{1}(\mathbf{r}, t)$, in the second system the density will be

$$
\rho_{2}(\mathbf{r}, t)=\frac{\rho_{2}^{*}}{\rho_{1}^{*}} \rho_{1}\left(\mathbf{r} \frac{L_{2}^{*}}{L_{1}^{*}}, t \frac{L_{2}^{*}}{L_{1}^{*}} \sqrt{\frac{p_{1}^{*} \rho_{2}^{*}}{p_{2}^{*} \rho_{1}^{*}}}\right) .
$$

This similarity was named in Ref. 14 the Euler similarity, and the parameter $v^{*} \sqrt{\rho^{*} / p^{*}}$ was named "the Euler number,' Eu. Note that this term is also used to designate the inverse square of our Euler number in the problem of a hydrodynamic flow past the body. ${ }^{8}$

\section{B. Shock waves}

It is very important that the Euler similarity covers not only smooth solutions of the Euler equations, but also solu- tions containing shock waves. Assume that in the system 1 there exists a shock wave. Then, if one chooses some other values of $L^{*}, \rho^{*}, p^{*}$, etc., with conditions (10) imposed, one finds a similarity solution for the system 2 in the zones before and after the shock. Now, one has to check that shock boundary conditions are invariant if constraints (10) are imposed. This will mean that solutions in the system 2 will be indeed similar to the solution in the system 1 , with the shock included. The invariance of the shock boundary conditions can be checked directly. Details can be found in Ref. 15. We therefore conclude that indeed the Euler similarity allows for the presence of the shocks.

\section{Strong drive}

There is a special case of a strong drive that can often be met both in the astrophysical objects (like SN explosions) and in corresponding laboratory experiments, and for which a very broad similarity holds. ${ }^{14}$ Assume that there is a system with an arbitrarily distributed initial density and pressure and initial velocities of the order of the sound velocities or less. Assume then that a planar (cylindrical, spherical) piston is moved into the system with a velocity much greater than the initial sound (and Alfvén) velocity. Considering as an example a spherical piston, we can describe its motion by the equation

$$
r=L^{*} q_{p}\left(\frac{t}{\tau^{*}}\right),
$$

where $\tau^{*}$ is the characteristic time of the piston motion (the time within which it is displaced by the distance $\sim L^{*}$ ); the dimensionless function $q_{p}$ (with subscript " $p$ " standing for the "piston") is of order of unity. The initial density distribution will as before be $\left.\rho\right|_{t=0}=\rho^{*} f\left(\mathbf{r} / L^{*}\right)$, with the function $f$ being of the order of unity.

The strong shock propagating in front of the piston brings the plasma to a new state; the characteristic pressure in this new state is

$$
p^{*} \sim \rho^{*} L^{* 2} / \tau^{* 2},
$$

and characteristic velocity is

$$
v^{*} \sim L^{*} / \tau^{*} .
$$

This state is essentially independent of either pressure, or velocity, or the magnetic field in front of the shock, provided they are small enough, as indicated at the beginning of this section (we will return to this issue at the end of this section).

If one takes the other system, with the scale factors $\tau^{*}$, $L^{*}$, and $\rho^{*}$ arbitrarily changed but with the functions $f$ in Eq. (6) and $q_{p}$ in Eq. (12) remaining the same (i.e., initial density distributions are geometrically similar, as are temporal dependencies of the piston position), the two systems behave similarly. For example, if in the system 1 characterized by scaling factors $L_{1}^{*}, \tau_{1}^{*}$, and $\rho_{1}^{*}$, the density is $\rho_{1}(\mathbf{r}, t)$, then in the system 2 the density will be

$$
\rho_{2}=\frac{\rho_{2}^{*}}{\rho_{1}^{*}} \rho_{1}\left(\mathbf{r} \frac{L_{2}^{*}}{L_{1}^{*}}, t \frac{\tau_{2}^{*}}{\tau_{1}^{*}}\right) .
$$




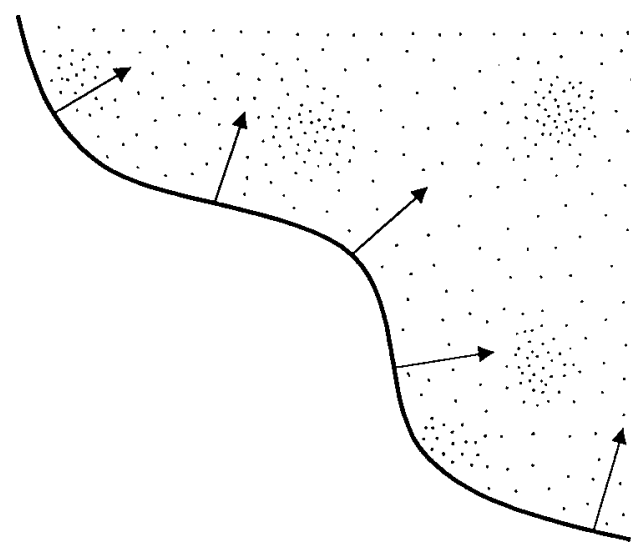

FIG. 1. A piston of an arbitrary shape moving into a cold nonuniform gas.

There is no need to impose either of the two constraints (10): the first is satisfied automatically, because of Eqs. (13) and (14); the second is unimportant because the magnetic field does not affect the strong shock (if, as we assume, the initial Alfvén velocity is much less than the shock velocity). In other words, all the characteristic parameters $\left(\tau^{*}, L^{*}, \rho^{*}\right.$, and $B^{*}$ ) can be varied independently, and still the similarity does exist. This very broad similarity can be extended to include the case where the piston surface deforms in an arbitrary fashion during the piston motion; to do that, one should just describe a piston by the general equation for a surface evolving with time: $F\left(\mathbf{r} / L^{*}, t / \tau^{*}\right)=0$, Fig. 1 . We still need the substance to be polytropic, at least behind the shock (for strong shocks, the shocked state depends only on the polytrope index of the shocked substance).

Although the magnetic field does not have a dynamical effect on the strong shock, its spatial structure behind the shock might be still interesting, e.g., in the problem of cosmic rays diffusion. To have the field structures be similar, it is sufficient to require that the initial magnetic field in the two systems obeys the last of Eqs. (6), with the same dimensionless function $\mathbf{k}$. The scaling factor $B^{*}$ can be chosen arbitrarily, with the only caveat that the field should be small in both systems, in the sense that the Alfvén velocity is much less than the piston velocity. If one wants not only the spatial structure but also the amplitude of the magnetic field to be scaleable, one should impose a constraint $B^{*} / \sqrt{\rho^{*}}=$ inv.

Finally, let us discuss a situation where the density and pressure nonuniformities in the initial state have the spatial scale, which is smaller than the distance $L^{*}$ that the piston travels within the time $\tau$. Let the length scale of the density distribution be $\alpha L^{*}$, with $\alpha<1$. What we need in order to apply the strong drive similarity is that the plasma in front of the shock would not change its spatial distribution prior to the shock arrival. This means that the initial sound and Alfvén velocities must be much less than $\alpha L^{*} / \tau$ (not just than the shock velocity $L^{*} / \tau$ ). If this condition is not satisfied, then we have to apply the general similarity of Sec. II A.

For the case of a cylindrical shock, what we call the strong-drive similarity has been established in numerical simulations of Ref. 17. A strong shock would fall on the rippled (in the $r-\theta$ plane) interface of two gases to produce a Richtmyer-Meshkov instability. At high-enough Mach numbers $(M>10)$ the shape of the perturbations taken at the same instant of a "dimensionless" time were independent of the Mach number.

\section{Varying composition}

So far, we have looked at the system with a uniform composition. It may, however, be interesting to consider a polytropic fluid of a varying composition. This would show itself up in that the coefficient $C$ in Eq. (3) will be now varying in space, following the varying composition. For the case of a short mean-free path, the mutual diffusion of the species can be neglected (see more details in Sec. IV), so that every parcel of the mixture is advected with its value of $\gamma$ remaining unchanged. This latter statement means that

$$
\frac{d \gamma}{d t} \equiv \frac{\partial \gamma}{\partial t}+(\mathbf{v} \cdot \nabla) \gamma=0 .
$$

The set of equations (1), (4) remains unchanged by allowing the adiabat index $\gamma$ to be a function of coordinates in the initial state; the further evolution of $\gamma$ is described by Eq. (16).

The similarity between two systems is preserved provided the initial distributions of $\gamma$ in both systems are geometrically similar. The case of an interface between two substances can be considered as a limiting case of the general problem, with a stepwise change of the coefficient $\gamma$ on the interface.

In the laboratory experiments it is common to use several layers of different materials to build an experimental package. It is therefore important that these materials could be described by power-law adiabats reasonably well, thereby approximating adiabat equations of an astrophysical problem.

\section{E. A SN explosion and the corresponding laboratory experiment}

As an example of applying aforementioned scaling considerations to a specific problem, we describe laboratory experiments directed toward simulation of the RayleighTaylor (RT) instability of the transitional zone between the helium and the hydrogen shell of the exploding type II supernova. To be specific, we consider the recent SN1987A, for which there exists a large amount of observational data, as well as results of numerous computer simulations (see, e.g., survey papers). ${ }^{18,19}$

The shock wave generated by the impulsive energy release in the core reaches the $\mathrm{He}-\mathrm{H}$ transition zone in about $500 \mathrm{~s}$. We are concerned with the hydrodynamic evolution of the transition zone after the passage of the shock. Because the hydrogen layer (situated outside the helium layer) has been strongly heated by the shock, the pressure gradient is directed outward, whereas the density gradient is directed inward (as helium is denser than the hydrogen). The effective gravitational acceleration, $g \equiv \nabla p / \rho$ is directed outward; it is many times greater than the real gravitational acceleration (directed inward). This is a canonical setting for the 
TABLE I. Comparing parameters of a SN 1987a plasma in the He-H transition layer and parameters of the laboratory experiment.

\begin{tabular}{llc}
\hline \hline Parameter & SN1987a & Laboratory experiment \\
\hline$L^{*}, \mathrm{~cm}$ & $9 \times 10^{10}$ & $5.3 \times 10^{-3}$ \\
$v^{*}, \mathrm{~cm} / \mathrm{s}$ & $2 \times 10^{7}$ & $1.3 \times 10^{5}$ \\
$\rho^{*}, \mathrm{~g} / \mathrm{cm}^{3}$ & $7.5 \times 10^{-3}$ & 4.2 \\
$p^{*}, \mathrm{dyn} / \mathrm{cm}^{2}$ & $3.5 \times 10^{13}$ & $6 \times 10^{11}$ \\
\hline \hline
\end{tabular}

Rayleigh-Taylor instability, which is thought to cause mixing of the material constituting various layers of supernova $^{20,21}$ and that is therefore of a significant interest for the laboratory experiment.

In the laboratory experiment, this situation is simulated by driving a shock wave through a package that contains an interface between copper and plastic layers. The shock comes from the copper side. Spatial distributions of plasma pressure and density at the time $t=2000 \mathrm{~s}$ in the SN and $t$ $=20 \mathrm{~ns}$ in the laboratory experiment are shown in Fig. 1 taken from Ref. 14 (simulations were performed by Kane). One sees that spatial distributions of the characteristic quantities look similar to each other. Imposing initial perturbations on the copper-plastic interface, one can then be confident that their further evolution will be similar in both systems (in the supernova, the perturbations are thought to appear because of a pre-existing convection).

Table I contains characteristic parameters of the two systems. We do not include the magnetic field, because its dynamic effect is thought to be negligible. We define the length scale $L^{*}$ as a width of the density peak at half-maximum, and the characteristic velocity as $L^{*}$. The characteristic density and pressure are taken in the middle points between two density jumps.

One can consider the states illustrated by Fig. 2 as initial states for the further evolution of the two systems. They are to a good accuracy geometrically similar. Constructing the Euler numbers for the two systems, one finds that they are close to each other (Table I). Therefore, according to the analysis of Sec. II A, the further evolution of the two systems will occur in a similar manner.

Creating a "good" initial state is probably the most challenging part of the laboratory experiment. The shock is driven by the ablation pressure from the irradiated side of the target. One has to adjust the composition and thicknesses of the layers constituting the experimental package, as well as the temporal dependence of the laser drive to achieve a "good" initial state. As soon as it has been created, the further evolution of the region near the interface will be similar to the evolution of the astrophysical counterpart during some time period, before hydrodynamic perturbations from the other interfaces (e.g., the rarefaction wave from the outer surface of the experimental package) return and violate the similarity. Despite the difficulty of this problem, there are many successful examples produced in the simulations (e.g., Ref. 22).
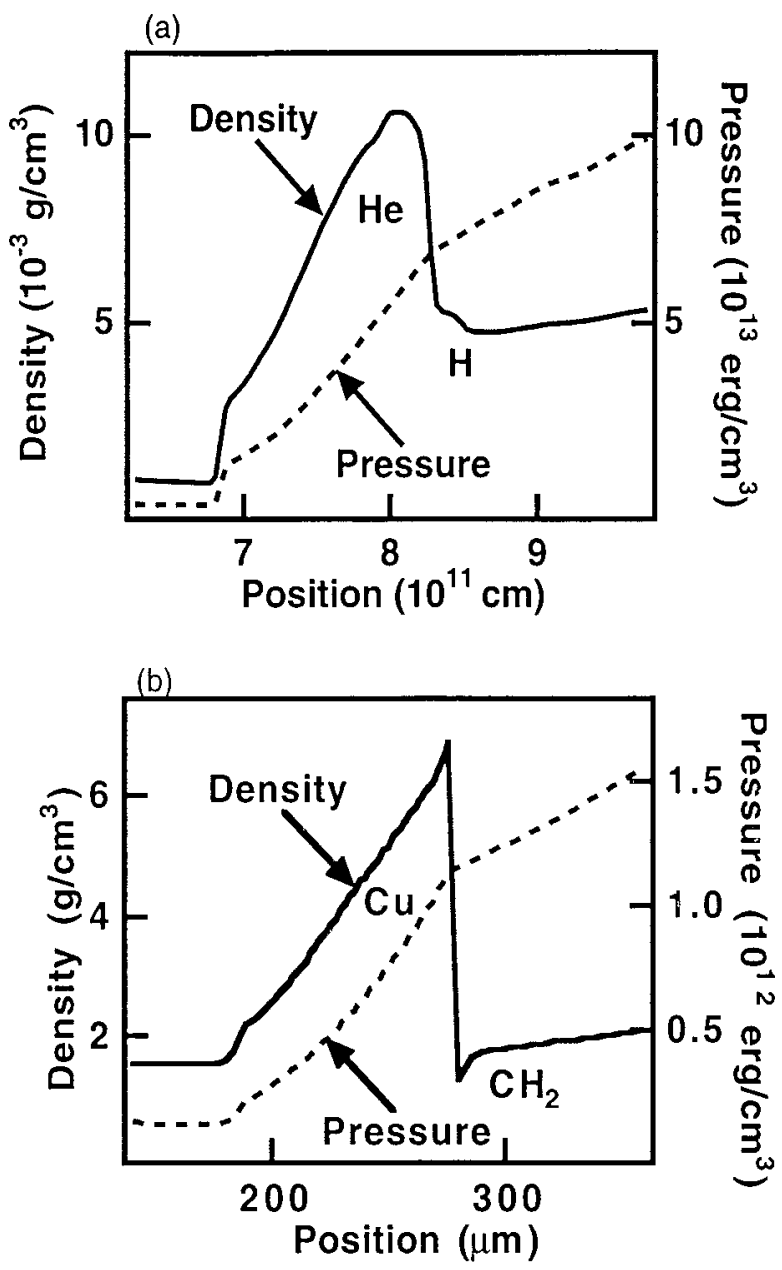

FIG. 2. Hydrodynamic solution for the supernova and the laboratory experiment: spatial profiles of the pressure and the density for the SN at $2000 \mathrm{~s}$ and the laboratory experiment at $20 \mathrm{~ns}$ (from Ref. 14).

\section{RADIATIVE LOSSES}

\section{A. Scaling considerations}

In the situations where the plasma is optically thin and, at the same time, radiation losses are significant on the time scale of hydrodynamic motion, the energy sink terms should be included explicitly into Eq. (4), which now becomes

$$
\frac{\partial p}{\partial t}+\mathbf{v} \cdot \boldsymbol{\nabla} p+\gamma p \boldsymbol{\nabla} \cdot \mathbf{v}=-(\gamma-1) Q_{\mathrm{rad}},
$$

where $Q_{\mathrm{rad}}=Q_{\mathrm{rad}}(p, \rho)$ is the power radiated per unit volume. If the medium becomes optically thick, then the heat transport has to be described by a diffusion-type equation. We return to the scaling in this case in Sec. IV.

For the problem described by Eq. (17), the difficulty in establishing similarity between the astrophysics and the laboratory lies in that, generally speaking, for an extremely broad range of variation of $p$ and $\rho$ that we encounter when scaling between astrophysics and the laboratory, the radiation power is some complex function of these arguments, not necessarily of a power-law type. If, however, $Q_{\text {rad }}$ can be approximated by a power-law function in some astrophysical object, and 
the same functional dependence could be reproduced in its laboratory analog, then establishing the similarity is straightforward. Indeed, for

$$
Q_{\mathrm{rad}}=A p^{\alpha_{1}} \rho^{\alpha_{2}}
$$

switching to dimensionless variables (8) converts Eq. (17) into

$$
\begin{aligned}
\frac{\partial \tilde{p}}{\partial \tilde{t}}+\tilde{\mathbf{v}} \cdot \tilde{\nabla} \tilde{p}+\gamma \tilde{p} \tilde{\boldsymbol{\nabla}} \cdot \tilde{\mathbf{v}} \\
\\
\quad=-(\gamma-1) A L^{*} p^{* \alpha_{1}-3 / 2} \rho^{* \alpha_{2}+1 / 2} \tilde{p}^{\alpha_{1}} \tilde{\boldsymbol{\rho}}^{\alpha_{2}} .
\end{aligned}
$$

Therefore, two systems behave similarly if, in addition to constraints (10), one more constraint is satisfied:

$$
A L^{*} p^{* \alpha_{1}-3 / 2} \rho^{* \alpha_{2}+1 / 2}=\text { inv. }
$$

We allow for the possible change of the coefficient $\mathrm{A}$ between two systems (power indices $\alpha_{1}$ and $\alpha_{2}$ must be the same).

The appearance of the additional constraint (20) reduces the freedom in selecting parameters of the laboratory experiment. Now only two of the parameters can be chosen arbitrarily, e.g., the spatial scale and the density. To keep the power indices $\alpha_{1}$ and $\alpha_{2}$ in the laboratory experiment as close as possible to those in an astrophysical object, one can adjust the composition of the matter in the laboratory experiment.

\section{B. Experiments with radiating jets relevant to astrophysics}

Astrophysical jets are generated by a variety of sources and cover many orders of magnitude in their spatial extent, from thousands of parsecs in case of galactic jets ${ }^{23}$ to a fraction of parsec in the case of jets originating from young nonstationary stars and associated with so-called HerbigHaro (HH) objects (e.g., Ref. 24). We discuss here experimental simulation of the latter group of jets. It is believed that these jets originate from nonstationary stars and plow into the ambient medium at a supersonic speed; radiative losses from the jet and shocked ambient plasma are believed to be an important term in the energy balance. Relevant theoretical analyses can be found, e.g., in Refs. 25 and 26.

Nonradiating jets have been studied in a great deal of detail in the laboratory (e.g., Ref. 27 and references therein). The first steps directed to generating radiative jets were reported in Refs. 28 and 29. In these two experiments radiating jets were generated by ablating hot plasma from the inner side of the conical surface, so that the ablated material would collapse on the cone axis and be ejected away from the apex. In the case of jets made of heavier elements, like $\mathrm{Au}$, the radiation from the dense core was significant, ${ }^{28,29}$ whereas for lighter materials, like Al, the radiation was weak. ${ }^{29}$ Just this fact shows the degree of control that experimentalists can exercise over the critical parameters that influence real astrophysical phenomena. The cooling parameter, defined as the ratio of the radiative cooling time for the jet core over the time within which jet advances by a distance equal to its radius, was in the range $\sim 1$ (for $\mathrm{Au}$ ), i.e., in the range corresponding to the value of this parameter in $\mathrm{HH}$ jets. In Ref.
29, a clear trend to generating narrower jets when using heavier, more strongly radiating materials was found, in agreement with the picture in which the on-axis implosion leads to a strong radiative cooling and corresponding stronger contraction for heavier materials.

One should note that jets generated in Refs. 28 and 29 were propagating essentially to a vacuum, whereas $\mathrm{HH}$ jets propagate into the ambient material with a non-negligible density (often even exceeding the jet density). The next step would be to add this ingredient to the laboratory experiment. To make experiments directly scaleable, one also needs to make sure that the cooling rate $Q_{\text {rad }}$ is a power-law function of $p$ and $\rho$. In the limited range of $p$ and $\rho$ covering an order of magnitude variation, a power-law approximation is a reasonable fit to cooling rate in $\mathrm{HH}$ jets. ${ }^{30}$ It has not been tried yet to match these power-law dependencies in the corresponding laboratory experiments; this may be possible by a proper choice of the jet composition and velocity (which sets the temperature scale).

In addition to laser experiments, radiative jets can be generated also in experiments with fast $\mathrm{Z}$ pinches (see the survey in Ref. 31). In one approach, one places a hole in one of the electrodes at the ends of the pinch, producing a jet by causing ejection of the pinch material through the hole (see Figs. 8 and 9 in Ref. 31). In another approach, ${ }^{7}$ one uses a conical wire array, causing the plasma that is blown off the wires to be assembled on axis and then ejected. This has been demonstrated to produce radiatively cooling jets of likely astrophysical relevance. ${ }^{7}$

\section{THE ROLE OF TRANSPORT PROCESSES}

\section{A. Collisionality}

A necessary condition for the validity of the equations of the ideal hydrodynamics is a sufficiently small mean-free path (mfp) of electrons and ions and, in the case where the radiation pressure is important, also of photons. The electron and ion mfp in a fully ionized plasma is determined by Coulomb collisions, whereas the photon mfp is determined by Compton scattering and inverse bremsstrahlung.

\section{Particle localization}

In some astrophysical objects the Coulomb mean-free path is greater than the characteristic length scale. For example, if one considers propagation of the blast wave from the SN explosion into a low-density plasma surrounding the star, one usually meets just this situation. In the case of SN 1987A the characteristic density and temperature at the distance of $3 \times 10^{16} \mathrm{~cm}^{-3}$ are $60 \mathrm{~cm}^{-3}$, and $3 \times 10^{4} \mathrm{eV}$, respectively. ${ }^{14}$ Therefore, the mean-free path is three orders of magnitude greater than the distance to the SN. At first sight, this precludes one from using the hydrodynamical description. Still, hydrodynamic equations are widely used in this case, because it is believed that the mean-free path is established by the random magnetic field. A very weak magnetic field is required to make the ion gyroradius orders of magnitude smaller than the characteristic length scale (e.g., a magnetic field of $1 \mu \mathrm{G}$ makes the $30 \mathrm{keV}$ proton gyroradius to be as small as $2.5 \times 10^{10} \mathrm{~cm}$ ). To designate this more gen- 
eral situation, where the mean-free path is determined by an entangled magnetic field, Drake has suggested the term "localization."14 When applying hydrodynamic equations we mean that localization is strong.

\section{Radiation entrainment}

When the radiation pressure is important, as it is, e.g., in the case of exploding $\mathrm{SN}$, the set of hydrodynamic equations (1) and (4) can be used only if radiation is strongly coupled with the matter. We briefly discuss here an issue of the radiation entrainment, assuming that, although the radiation pressure may be dominant, the mass density of the system is still determined by the ions. This implies that $\rho$ entering equations (1) is the particle mass density, not the equivalent radiation mass density $4 \sigma T^{4} / c^{3}$, where $\sigma$ is the StefanBoltzmann constant; the opposite case would correspond to very low particle mass densities. A number the astrophysical objects mentioned in the Introduction satisfy this assumption.

For a short enough mean-free path of the photons, the radiation is strongly coupled to the matter so that the average velocity of the photons in the Planckian distribution is equal to the hydrodynamic velocity $\mathbf{v}$ of the particles. Accordingly, if a certain parcel of the gas is, say, compressed, the radiation filling this parcel is also dragged into compression and the radiation pressure merely follows Eq. (4) with $\gamma=4 / 3$ (because, for the radiation, $p=\epsilon / 3$ ). In the case of the $\mathrm{He}-\mathrm{H}$ transition layer in the SN 1987a, a typical photon mean-free path (determined in this case by Compton scattering) is less than $10^{3} \mathrm{~cm}$, whereas the characteristic spatial scale of, say, density variation is $\sim 10^{11} \mathrm{~cm}$. The coupling is, therefore, very strong and the use of the Euler equations with $\gamma=\frac{4}{3}$ is well justified.

\section{B. Transport of heat, momentum, and matter}

Normally, the condition that the mean-free path is very small compared to the length scale of the problem means also that the transport processes are slow and cause only a small correction to the ideal magnetohydrodynamics (MHD). Consider, for example, the effect of the thermal conduction. The time for the heat to spread over the area with the characteristic length scale $L^{*}$ is on the order of $L^{* 2} / \chi$, where $\chi$ is the thermal diffusivity. The characteristic time of the hydrodynamic motions in the initial value problem is $L^{*} \sqrt{\rho^{*} / p^{*}}$ (in the initial value problem for the compressible fluid, it is natural to assume that the characteristic velocity is of the order of the sound speed). The ratio of the two forms a dimensionless number (the Peclet number):

$$
\mathrm{Pe}=\frac{L^{*} \sqrt{p^{*} / \rho^{*}}}{\chi} .
$$

If the Peclet number is large, the effect of the thermal diffusivity is small. For the viscous momentum transport the same role is played by the familiar Reynolds number. In the initial value problem, the velocity field varies with time. We choose as a characteristic velocity the quantity $\sqrt{p^{*} / \rho^{*}}$, which is of the order of the sound speed. Accordingly, the Reynolds number is

$$
\operatorname{Re}=\frac{L^{*} \sqrt{p^{*} / \rho^{*}}}{\nu}
$$

The role of the interspecies diffusion can be characterized by a dimensionless parameter $\left(L^{*} / D\right) \sqrt{p^{*} / \rho^{*}}$, analogous to (21) and (22), with $D$ being the interspecies diffusion coefficient. This parameter is sometimes called the Peclet mass number and denoted as $\mathrm{Pe}_{m}$.

Transport coefficients $\chi, \nu$, and $D$ are proportional to the mean-free path. So, indeed, the smallness of the mean-free path would usually mean an insignificant role of transport processes (i.e., large values of $\mathrm{Pe}$ and $\mathrm{Re}$ ).

\section{Small spatial scales}

It would be, however, premature to assume that, if the plasma is strongly collisional, transport processes can be universally neglected. In a number of cases, the motion on the scale much smaller than $L^{*}$ becomes important. This may occur in the case of instabilities of sharp interfaces, or when the system becomes strongly turbulent and develops smallscale vortices through the cascading process. We will discuss this issue in the context of viscous effects in the RayleighTaylor instability of accelerated fluid in the exploding supernova, in the transition layer between the helium and hydrogen shells, whose thickness sets the characteristic scale $L^{*}$ of this specific problem. An analysis of viscous effects in a broad variety of settings can be found in Refs. 32 and 33.

We assume that the unperturbed motion is a spherically symmetric radial expansion. The characteristic velocity of this motion is $\sqrt{p^{*} / \rho^{*}}$ and the characteristic time scale is

$$
\tau^{*} \sim L^{*} \sqrt{\rho^{*} / p^{*}} .
$$

Dividing the first by the second, one finds the characteristic acceleration $a^{*} \sim p^{*} / \rho^{*} L^{*}$. Then, the perturbation with a length scale $\chi \ll L^{*}$ has a growth rate $\Gamma \sim \sqrt{a^{*} / L^{*}}$. The characteristic time of viscous dissipation on the scale $\chi$ is $\tau_{\text {visc }}$ $\sim \chi^{2} / \nu$. The viscosity will strongly affect perturbations with $\Gamma \tau_{\text {visc }}<1$, i.e., perturbations with the length scale smaller than some critical value $\chi_{\text {visc }}=\left(L^{*} \nu^{2} / a^{*}\right)^{1 / 4}$. Recalling the expression for $a^{*}$ and using the definition (22) for the Reynolds number, one can also present the expression for the critical scale as

$$
\chi_{\text {visc }}=L^{*} / \operatorname{Re}^{1 / 2} \text {. }
$$

Therefore, even if the Reynolds numbers in both the astrophysical object and its laboratory counterpart are large, the relative value of the scale where the model of the ideal fluid breaks down may be different (unless the Reynolds numbers are just equal, which seems to be not very probable). Note, however, that for the values of Reynolds numbers mentioned in Sec. II E the viscous effects appear only at very short scales, orders of magnitude smaller than the global scale.

Consider now larger-scale perturbations, with the length scale approaching $L^{*}$. Such perturbations are thought to play the most important role in the gross mixing of the fluid. At the linear stage of their growth, viscous effects are negligible. However, when the perturbation reaches a strongly 
nonlinear stage, with a well-developed structure of bubbles and spikes, shear flows with relative velocity of order of the characteristic velocity $\sqrt{p^{*} / \rho^{*}}$ develop within the time $\sim \tau^{*}$ [Eq. (23)]. The Reynolds number for this flow is roughly equal to the global Reynolds number (22) and is typically much higher than the critical Reynolds number for the onset of the shear flow instability. After several turnaround times of large-scale vortices, i.e., after a few $\tau^{*}$, smaller-scale vortices appear. If one assumes the Kolmogorov model of the turbulence, the vortices with some scale $\chi$ appear within the time $\sim \tau^{*} \ln \left(L^{*} / \chi\right)$. Viscous dissipation "turns on" when the scale $\chi$ reaches the dissipation scale, which is (e.g., Ref. 34)

$$
\chi_{\text {diss }} \sim L^{*} / \mathrm{Re}^{3 / 4} .
$$

This takes time $\sim \tau^{*} \ln \mathrm{Re}$, which is in the examples considered in Sec. IIE roughly speaking an order of magnitude longer than the characteristic time $\tau^{*} \sim L^{*} \sqrt{\rho^{*} / p^{*}}$.

In other words, the Euler similarity (with dissipative terms neglected) will correctly describe an early stage of the instability, until small-scale vortices with $\chi \sim \chi_{\text {diss }}$ are formed. During this early stage there is no need to make any assumptions about the turbulent viscosity, introduce Reynolds stresses, and other approximate ways of description: the Euler equations correctly describe this stage, including the formation of smaller-scale vortices. In a number of cases, including the SN explosion and its laboratory simulation, this means that essentially the whole physical process is correctly described by Euler equations, with viscosity neglected: within the time $\sim \tau^{*} \ln$ Re the system already reaches a very different state of a strong mix.

It is interesting however to assess an issue of what would happen later in time, were there a need to study this later evolution. The question is to what extent will behavior of the two systems be similar at the larger scales if Reynolds numbers [defined according to Eq. (22)], though very large, are different in the two systems (meaning that relative values of the dissipative scales are different). One can argue that the differences on the global scale will be not very large; they will probably appear in the terms of order of $\ln \mathrm{Re}$, or in terms that depend on Re even weaker. The reason for this hypothesis is that in a number of relevant problems exhaustively studied experimentally the situation is just this. Examples include the turbulent pipe flow (e.g., Ref. 34) and a turbulent flow past a body at high Reynolds number (see Refs. 35-37 for excellent pictures of such flows in a broad range of Reynolds numbers).

In the case of a compressible turbulence, where the kinetic energy of the gas is comparable to its thermal energy, the dissipation produced in small vortices has to be retained in the energy equation. Indeed, the energy equation with the viscous dissipation taken into account takes the form

$$
\frac{\partial p}{\partial t}+\mathbf{v} \cdot \boldsymbol{\nabla} p+\gamma p \boldsymbol{\nabla} \cdot \mathbf{v}=Q_{\mathrm{visc}},
$$

$$
\begin{aligned}
Q_{\mathrm{visc}}= & (\gamma-1) \cdot\left[\frac{\nu \rho}{2}\left(\frac{\partial v_{i}}{\partial x_{k}}+\frac{\partial v_{k}}{\partial x_{i}}-\frac{2}{3} \delta_{i k} \frac{\partial v_{l}}{\partial x_{l}}\right)^{2}\right. \\
& \left.+\nu_{1} \rho(\boldsymbol{\nabla} \cdot \mathbf{v})^{2}\right],
\end{aligned}
$$

where, for completeness, we have introduced also the bulk viscosity $\nu_{1}$, which for the gases is usually of the order of $\nu$. One can check that, for well-developed turbulence with a Kolmogorov spectrum, the main contribution to the viscous dissipation comes from the scales determined by Eq. (25). Assuming that velocity at the global scale is of the order of sound velocity $s$, one finds that the velocity $v_{\text {diss }}$ at dissipative scales is of the order of $s\left(\chi_{\text {diss }} / L^{*}\right)^{1 / 3}$. Estimating the viscous terms on the rhs of Eq. (26) as $Q_{\text {visc }}$ $\sim \nu \rho\left(v_{\text {diss }} / \chi_{\text {diss }}\right)^{2}$, one finds that, not surprisingly for the developed turbulence, $Q_{\text {visc }}$ does not depend on the viscosity or the Reynolds number: $Q_{\text {visc }} \sim \rho s^{3} / L^{*}$. This simple estimate supports our hypothesis that the large-scale features of the motion are only weakly affected by the Reynolds number, provided it is very large.

This is not to say that small-scale vortices cannot have a significant global effect. One possible example is the molecular mixing. If one has a system where the elemental composition varies on the global scale, the mutual diffusion over the global scale would take a very long time, roughly speaking, the mass Peclet number (Sec. IV B 2) times the hydrodynamic time $L^{*} / s$. The turbulence brings parcels of fluid with a different composition close to each other, and diffusion smoothes the composition within a very short time, ${ }^{34,38}$ of the order of a few hydrodynamic turnaround times. This is particularly important in the systems with reacting components, where they were initially spatially separated. The other example is molecular mixing of the reacting material and reaction products, which may lead to a decrease of the overall reaction rate. The latter example is relevant to the physics of type I supernovae explosions, which are believed to be driven by thermonuclear burn. ${ }^{39}$ All in all, in the discussion of this section we show that the issues of the role of smaller scales need a careful approach.

\section{Magnetic diffusion}

In astrophysical plasmas the parameter beta, $\beta$ $=8 \pi p / B^{2}$, which measures the ratio of the plasma pressure to the magnetic pressure, can vary in a broad range. For example, in our Sun beta is typically much greater than 1 in the solar interior, and is less than 1 in the upper chromosphere and lower corona. In more distant objects one can also meet both $\beta>1$ and $\beta<1$ cases. If there occurs a threedimensional (3-D) contraction of a conducting medium driven by gravitational forces [or photoablative pressure, as is the case in some gaseous nebulas (Sec. V B)], the density scales as $1 / r^{3}$, and the pressure of a monatomic gas scales as $\left(1 / r^{3}\right)^{5 / 3}=1 / r^{5}$. Conservation of the magnetic flux yields $1 / r^{2}$ dependence of the magnetic field and $1 / r^{4}$ dependence of the magnetic pressure. Therefore, at a high-enough compression ratio the gaseous pressure becomes higher than the magnetic pressure, and the $\beta>1$ case is realized. Compression of the gas by a strong shock also favors the generation 


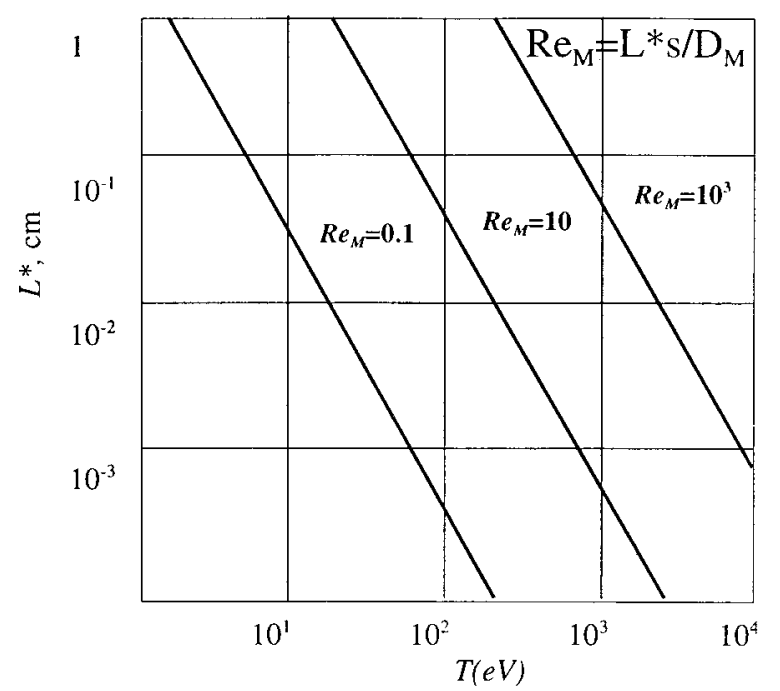

FIG. 3. Lines $\operatorname{Re}_{M}=$ const for a fully stripped carbon in the $L^{*}-T$ plane.

of a high- $\beta$ plasma because the magnetic field increases in proportion with density and remains finite even at the infinite Mach number, while the gaseous pressure in a strong shock increases as the square of the Mach number. On the other hand, if the gas strongly radiates and loses the thermal energy, the magnetic pressure may become dominant $(\beta<1)$.

Experiments with high-intensity lasers provide an opportunity for the studies of a high- $\beta$ plasma, because of the high plasma densities involved. By immersing an experimental package into a bias magnetic field, one can create a welldefined initial situation, which would then evolve according to Eqs. (1) and (4).

The third equation of the set (1) corresponds to a perfectly conducting plasma, i.e., to a perfect line tying, when the magnetic field lines are "glued" to liquid particles. Finite plasma resistivity leads to the appearance of the magnetic diffusion. The magnetic diffusion coefficient, $D_{M}$, is related to the plasma resistivity, $\eta$, by the equation $D_{M}$ $=c^{2} \eta / 4 \pi$. The arguments identical to those used in the discussion of viscous friction and thermal conduction in Sec. IV B show that the magnetic diffusion is small if the dimensionless parameter,

$$
\operatorname{Re}_{M} \equiv s L^{*} / D_{M},
$$

which we call the magnetic Reynolds number, is much greater than 1 . Note that in this definition, as well as in the definition of the Reynolds and Peclet numbers, we use the sound speed $s$ as a characteristic velocity.

Under the astrophysical conditions, the magnetic Reynolds number is extremely large, due to very large spatial scales involved. For example, for the hydrogen plasma with the temperature $\sim 0.1 \mathrm{eV}$, at the scale typical for the HerbigHaro jets $\left(L^{*} \sim 10^{17} \mathrm{~cm}\right)$, it is of the order of $10^{15}$. Reaching such values of $\mathrm{Re}_{M}$ in the laboratory experiments with laserdriven targets is very difficult if not impossible. On the other hand, reaching the values $\operatorname{Re}_{M} \gg 1$ is possible (Fig. 3). A key parameter is the plasma temperature. Its increase obviously is facilitated by using more powerful lasers. Note that the collisional mean-free path in these experiments is very short,

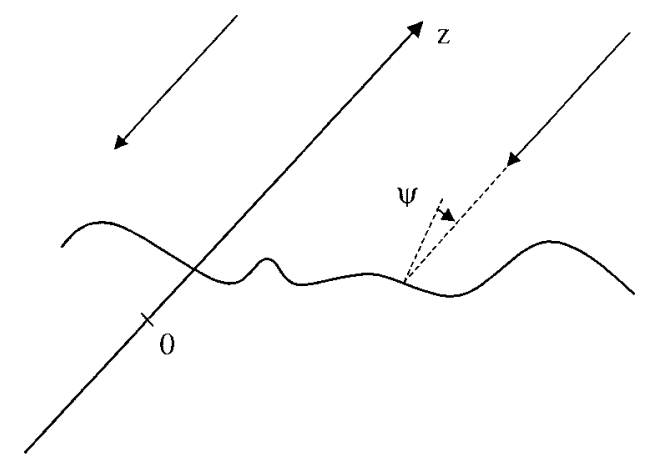

FIG. 4. Geometry of the photoevaporation front (boldfaced curved line). Thin arrows directed oppositely to the axis $z$ depict the incident radiation, $\psi$ is the angle between the local normal to the photoevaporation front and the direction of radiation.

and the density is high, so that the Ohm's law can be used in its simplest single-fluid version, $\mathbf{j}=\sigma(\mathbf{E}+\mathbf{v} \times \mathbf{B} / c)$. This is a unique feature of high-energy density experiments compared to their magnetic fusion energy (MFE) counterparts. Among specific problems that might be studied in such experiments are magnetic reconnections and a magnetic dynamo in highbeta regimes. Lower-beta regimes of reconnection are more easily accessible for MFE-type devices, which have already been successfully used for this purpose (e.g., Ref. 40).

\section{SIMILARITIES IN THE EVOLUTION OF ABLATION FRONTS}

\section{A. Scaling for the ablatively driven hydrodynamics}

When intense radiation falls on a boundary of semispace filled with the radiation-absorbing matter, evaporation/ ionization of the surface layers begins, and a gas flow directed away from the surface forms (ablation flow). Because of a "rocket effect," the matter in front of the ablation zone experiences acceleration in the opposite direction. This setting can be met in a number of astrophysical problems, in particular, in the problem of photoevaporated molecular clouds. ${ }^{41}$ Among many physical effects accompanying photoevaporation, there is Rayleigh-Taylor (RT) instability of the ablation front: ${ }^{41,42}$ since the density of the ablated material is smaller than the initial density of the matter, one finds here a classical setting for this instability in the frame comoving with the ablation front (the effective gravity acceleration $g$ is directed from the heavy to the light fluid).

We discuss the scaling laws in the ablation front problem for one of the possible models of radiation absorption. Assume that the incident radiation at a large distance from the ablation front forms a plane-parallel flow and choose the propagation direction as axis $z$ (directed against the radiation flow, Fig. 4). We do not assume that the averaged boundary is normal to the direction $z$. We assume that the time for establishing the ionization equilibrium in any particular volume is short compared to the characteristic time of the hydrodynamic motion. Then, the extinction coefficient depends on the instantaneous values of the temperature and density, or, equivalently, on the instantaneous values of the pressure 
and density: $\kappa=\kappa(p, \rho)$. Simple scaling is possible if $\kappa$ has a power law dependence on its arguments,

$$
\kappa=A_{\text {abs }} p^{\zeta_{1}} \rho^{\zeta_{2}} \text {. }
$$

The power $Q_{\text {abs }}$ absorbed per unit volume is $Q_{\text {abs }}=\kappa I$, where $I$ is the radiation intensity. It is convenient to normalize $I$ to the intensity $I_{\infty}$ at a large distance from the ablation front, at $z \rightarrow \infty, I=J I_{\infty}$, where $J$ is a dimensionless intensity. The intensity $I_{\infty}$ generally speaking, depends on time, so that

$$
I_{\infty}=I_{\infty}^{*} F\left(\frac{t}{\tau^{*}}\right),
$$

where $F$ is a dimensionless function of order one, and $I_{\infty}^{*}$ and $\tau^{*}$ are normalizing factors. The equation for $J$ reads as

$$
\frac{d J}{d z}=A_{\mathrm{abs}} p^{\zeta_{1}} \rho^{\zeta_{2} J}
$$

The boundary condition is that $J$ becomes 1 at $z \rightarrow \infty$, where absorption vanishes.

Hydrodynamic equations (1) do not change, whereas the entropy equation (4) becomes

$$
\frac{\partial p}{\partial t}+\mathbf{v} \cdot \boldsymbol{\nabla} p=-\gamma p \boldsymbol{\nabla} \cdot \mathbf{v}+I_{\infty} J A_{\mathrm{abs}} p^{\zeta_{1}} \rho^{\zeta_{2}} .
$$

Introducing dimensionless variables (8) into Eqs. (30) and (31), one finds that two systems would be described by the same set of dimensionless equations if three additional [to Eq. (10)] conditions are satisfied, namely

$$
\begin{aligned}
& \frac{\tau^{*}}{L^{*}} \sqrt{\frac{p^{*}}{\rho^{*}}}=\text { inv, } \quad A_{\mathrm{abs}} L^{*} p^{* \zeta_{1}} \rho^{* \zeta_{2}}=\mathrm{inv} ; \\
& \frac{I_{\infty}}{p^{*}} \sqrt{\frac{\rho^{*}}{p^{*}}}=\text { inv. }
\end{aligned}
$$

One sees that the system becomes significantly more constrained than the set of Euler equations alone.

To make things worse, the model described by Eqs. (29)-(31) misses two potentially significant physical effects. First, it does not include effects related to the change of the ionization state of the gas and, therefore, does not describe the corresponding deviations from the polytropic equation of state. Second, it ignores the fact that the ionizing radiation, generally speaking, has a broad spectrum; each spectral interval has its own function $\kappa=\kappa(p, \rho)$, so that the system immediately becomes overconstrained. Still, even a simple model (29)-(31) catches gross features of the phenomenon and should correctly describe its overall morphology.

\section{B. A model with strong photoabsorption and large density contrast}

There are situations, however, where the realistic scaling is possible without introducing too many additional constraints. Those are the situations where the characteristic absorption length of the radiation, $1 / \kappa$, is much less than the other spatial dimensions, and where, simultaneously, the ionized ablation flow has a small density and is transparent to the incoming radiation. In the case of the Eagle nebula, for example, taking as a characteristic initial density of the mo- lecular hydrogen the value of $5 \times 10^{4} \mathrm{~cm}^{-3}$, and noting that the characteristic photoabsorption cross section is $\sim 10^{-18} \mathrm{~cm}^{-3}$, one finds that the absorption occurs at the scale $\sim 2 \times 10^{13} \mathrm{~cm}$, which is four to five orders of magnitude smaller than the characteristic dimensions of the initial cloud (see Refs. 43-45 for more information). Also, the density of the ablated material is at least 50 times less than the cloud density, and it is almost fully ionized (this is what makes so strong a drop in the absorption coefficient). A density contrast of 50 could severely test numerical codes.

Considering a cloud with a sharp boundary, one can say that the ablation pressure is acting on this boundary. The velocity at which the ablation front erodes the cloud is very slow compared to the velocity of the shock launched into the cloud, and can be neglected. ${ }^{46}$ (This, by the way, makes negligible the ablative stabilization of the RT modes of interest for the Eagle nebula evolution). ${ }^{46}$ Therefore, we arrive at the problem where the effect of ablation can be described as the ablation pressure acting on the boundary of the cloud. In the case of the Eagle nebula, the ablation pressure is much higher than the initial pressure of the molecular gas, so that the "strong drive" case can be applied. Let us approximate the ablation pressure as some power-law function of the incident radiation flux,

$$
P_{\mathrm{abl}}=A_{\mathrm{abl}}\left(\cos \psi I_{\infty}\right)^{\zeta_{3}},
$$

where $\psi$ is an angle between the axis $z$ and the local normal to the surface (Fig. 4). In a strong-drive case, initial pressure and initial velocity can be considered as negligibly small. Then, following the line of reasoning identical to the one described in Sec. IIC, one concludes that the similarity between two systems requires imposing of only one constraint,

$$
\frac{\tau^{*}}{L^{*}} \sqrt{\frac{p_{\mathrm{abb}}^{*}}{\rho^{*}}}=\mathrm{inv},
$$

where $p_{\mathrm{abl}}^{*} \equiv A_{\mathrm{abl}}\left(I_{\infty}^{*}\right)^{\zeta_{3}}$, and $\tau^{*}$ is the parameter that enters Eq. (29) and characterizes the temporal dependence of the incident radiation. If condition (34) holds between two systems, the astrophysical system and its laboratory counterpart, then all the other parameters of these two systems are related just by simple scale transformations, e.g., the pressure scales as $p_{\text {abl }}^{*}$, the characteristic time scales as $L^{*} \sqrt{\rho^{* / p_{\text {abl }}^{*}}}$, etc. It goes without saying that the initial density distributions should be geometrically similar.

In the astrophysical photoevaporation problems, one can meet a situation where the shock-heated material of the molecular cloud quickly loses energy due to radiative cooling, mostly in rotational molecular transition in the millimeter range (e.g., Ref. 47). In such cases additional constraints of the type discussed in Sec. III A have to be imposed. In what follows, we assume that radiative losses from the cloud interior are insignificant.

In Table II, we present characteristic values of the parameters typical for the Eagle nebula cloud, and those of a possible laboratory experiment. The density in the laboratory experiment is that of a typical plastic. The parameters of the laboratory experiment are chosen in such a way as to make the dimensionless number (34) equal in both systems. One 
TABLE II. Comparing parameters of the Eagle nebula and parameters of a possible laboratory experiment.

\begin{tabular}{lcc}
\hline \hline \multicolumn{1}{c}{ Parameter } & Eagle nebula & Possible laboratory experiment \\
\hline$L^{*}, \mathrm{~cm}$ & $10^{18}$ & $6 \times 10^{-3}$ \\
$p_{\mathrm{abl}}^{*}, \mathrm{dyn} / \mathrm{cm}^{2}$ & $5 \times 10^{-9}$ & $10^{13}$ \\
$\rho^{*}, \mathrm{~g} / \mathrm{cm}^{3}$ & $1.5 \times 10^{-19}$ & 1.3 \\
$\tau^{*}, \mathrm{~s}$ & $6 \times 10^{12}$ & $2.4 \times 10^{-9}$ \\
\hline \hline
\end{tabular}

has of course to check whether the adiabat with $\gamma=\frac{7}{5}$ is a good approximation for the material used in the laboratory experiment.

Fortuitously, there already exist data from laser ablation experiments obtained in the regimes not very different from those presented in Table II. ${ }^{48}$ An exhaustive study of the radiation transport and ablation front structure for conditions close to those of Ref. 7 have been carried out in Ref 49. In experiments ${ }^{48}$ the density of the ablated material was indeed small compared to the density of the initial foil. Absorption in these experiments occurs in a relatively narrow layer, as required by our model. Ablative stabilization ${ }^{4,50}$ plays a role only for short wavelengths, $x<6 \mu \mathrm{m}$, whereas the range of wavelengths studied was up to $X=15 \mu \mathrm{m}$. It is interesting to note that the structure of well-developed RT perturbations observed in Ref. 48 is very similar to the so-called "elephant trunks" observed in the Eagle nebula [see Fig. 2(c) of that paper and Fig. 2 of Ref. 44].

There is a special case of the similarity considered in this subsection: the case where the radiation flux turns on in a stepwise manner at $t=0$, and then stays constant. In this case, even constraint (28) disappears, and all the parameters of the simulation experiment (of which there remain only three, $p_{\mathrm{abl}}^{*}, \rho^{*}$, and $L^{*}$ ) can be chosen arbitrarily. The temporal scale of the process is defined by the second equality in Eq. (8), with $p^{*}$ replaced by $p_{\mathrm{abl}}^{*}$.

In Eq. (33) we have retained the dependence of the energy flux (and, accordingly, of the ablation pressure) on the angle $\psi$ between the normal to the surface and the direction in which radiation propagates. This dependence, for $\psi \sim 1$, gives rise to strong modification of the RT instability: ${ }^{51}$ the growth rate of surface ripples becomes strongly dependent on the propagation direction relative to the plane of incidence; unlike the RT instability, ripples propagate with a finite phase velocity along the surface. This modified instability was called in Ref. 51 "Tilted Radiation," or TR instability. For wavelengths shorter than the thickness of the flyer plate $\left(k L^{*}>1\right)$ the growth rate $(\operatorname{Im} \omega)$ and the frequency $(\operatorname{Re} \omega)$ of the TR instability are determined by simple expressions:

$$
\begin{aligned}
& \operatorname{Im} \omega=\sqrt{\frac{g k}{2}} \sqrt{1+\sqrt{1+L^{* 2} k^{2} \sin ^{2} \psi \cos ^{2} \vartheta}}, \\
& \operatorname{Re} \omega=\sqrt{\frac{g k}{2}} \sqrt{\sqrt{1+L^{* 2} k^{2} \sin ^{2} \psi \cos ^{2} \vartheta}-1},
\end{aligned}
$$

where $\vartheta$ is the angle between the wave vector of the perturbation and the plane of incidence. Reference 51 speculates that, in the nonlinear stage, this instability may lead to a formation of blobs of a matter detached from the surface of the cloud and traveling along the surface with the velocity approximately equal to the phase velocity. The TR instability can be scaled from astrophysical objects to laboratory experiments, provided the condition (34) holds.

\section{CONCLUSION}

We have shown that there is a broad class of astrophysical objects that can be adequately simulated in high-energydensity laboratory experiments. Those include exploding type II supernovae, nonradiating and radiating jets, and photoevaporation fronts. A common feature of these phenomena is that they can be adequately described by hydrodynamic equations, with thermal conductivity, viscosity, and mutual diffusion of various species neglected. Shock waves are allowed, as well as nonuniformities of the composition. If radiative losses are negligible, then the similarity conditions are very nonrestrictive, allowing one to choose from a broad variety of parameters of a possible simulation experiment. The main constraint (which is usually met in the highenergy-density experiments) is that the matter should indeed be strongly collisional and obey magnetohydrodynamic equations. We call the corresponding similarity "the Euler similarity." Adding the radiation leads to a more constrained system but still some flexibility remains.

The role of viscosity, thermal diffusivity, and particle diffusivity can be conveniently expressed in terms of dimensionless numbers, the Reynolds number, the Peclet number, and the magnetic Reynolds number. All of them are typically very large in the astrophysical systems, meaning the negligible role of dissipative processes for large-scale motions. If, however, small-scale motions are for some reason important, one should carefully analyze them, to see if dissipative processes are still unimportant. In the case where the dynamical evolution of the system reaches the stage of a strongly developed hydrodynamic turbulence, with a Kolmogorovtype spectrum established, the viscous dissipation certainly comes to play for small-scale vortices. We, however, have presented arguments that show that, for very large Reynolds numbers, two systems would behave very similarly at the global scale, even if the Reynolds numbers are not equal (say, $10^{10}$ and $10^{6}$ ). Moreover, in a number of situations (e.g., when considering the process of the RT spikes poking through the photosphere of a supernova), one does not need to track the evolution of the system to the state of fully developed turbulence; it is sufficient to follow its evolution for a couple of turnaround times of large-scale vortices. In such a case the Euler similarity works in its direct form.

Simulation experiments carried out so far demonstrate that indeed various aspects of astrophysical phenomena can be simulated in a scaled fashion. Remarkable progress has been achieved in the studies of hydrodynamics of SN type II explosions, both at the early stage, before the shock breakout through the photosphere, and at the stage when ejecta begin to interact with the stellar wind and interstellar medium (see Ref. 1 and references therein). It has recently been shown ${ }^{52}$ that, for the $\gamma=\frac{5}{3}$ adiabat, there exists a similarity between an 
explosion and an implosion; this would allow one to simulate astrophysical explosive events in the gas with $\gamma=\frac{5}{3}$ in implosions of pellets in IFE experiments.

Radiative jets with parameters similar to the ones met in astrophysical objects have been reproduced. First experiments involving jets from $\mathrm{Z}$ pinches began. Great potentialities of experimental simulations of the photoevaporating molecular clouds have been identified. Some of the earlier experiments, whose goal was not related to astrophysical simulations, have, in fact, been carried out in the regimes of interest for scaled simulations.

There are many other objects in the sky whose MHD behavior could be simulated in the experiments with intense lasers and fast $\mathrm{Z}$ pinches. We list here a few examples (some of which are speculative).

(1) It would be important to simulate current-carrying astrophysical jets, as well as magnetic reconnection and magnetic dynamo. Lasers and $\mathrm{Z}$ pinches are well suited for the production and study of plasmas with high beta, and may prove able to study certain MHD effects in such plasmas. On the other hand, low-beta effects are more suitable for the studies in MFE devices.

(2) Rotation is generally a very important factor in astrophysics. There are ways that allow one, in principle, to spin the spherical pellets up in laser experiments. ${ }^{53}$ Developing this technique could provide a better understanding of the SN physics.

(3) In the physics of type I supernovae, a very important factor is a proper description of hydrodynamics of a reacting matter (fusion reactions are believed to be the source of energy for type I supernovae). This problem may become accessible for the laboratory simulations when significant fusion reaction rates will be achieved in the future IFE experiments.

(4) The most challenging problem is that of simulating gravitational effects arising from the mutual attraction of different parcels of the gas and leading, e.g., to the Jeans instability. In the laboratory experiments, mutual gravitational attraction is extremely small. It may, however, be possible to simulate gravitational effects by a judicious use of the effects of ionization-recombination thermal instabilities, where the matter coalesces to higher-density structures. This phenomenon is known for decades in a gas discharge plasma (e.g., Ref. 54). Similar effects have recently been reported in laser produced plasmas. ${ }^{55}$ If the basic equations describing such processes are similar to the hydrodynamic equations of gravitating matter, at least up to the density contrast of 10 or so, it may become possible to experimentally address issues of the global structures ${ }^{56}$ formed in the Universe by the Jeans instability (is the "cellular" structure actually present?).

This list is by no means complete. There exists a number of other exciting objects and processes in the Universe that may lend themselves to laboratory simulations.

\section{ACKNOWLEDGMENTS}

The authors are grateful to D. Arnett, J. Blondin, J. Castor, D. Dearborn, J. Glimm, D. Hill, W. Hsing, J. Kane, R. Klein, J. Knauer, E. Liang, R. McCray, M. Pound, R. Rosner, J. Stone, and S. Woosley for helpful discussions. This work was performed for the U.S. Department of Energy by UC LLNL under Contract No. W-7405Eng-48.

${ }^{1}$ B. A. Remington, R. P. Drake, H. Takabe, and D. Arnett, Science 284, 1488 (1999); B. A. Remington, R. P. Drake, H. Takabe, and D. Arnett, Phys. Plasmas 7, 1641 (2000).

${ }^{2}$ R. P. Drake, J. Geophys. Res. 104, 515 (1999).

${ }^{3}$ B. A. Remington, J. Kane, R. P. Drake et al., Phys. Plasmas 4, 1994 (1997).

${ }^{4}$ H. Takabe, H. Nagamoto, A. Sunahara, N. Ohnishi, A. I. Mahdy, Y. Yoda, S. Naruo, H. Azechi, H. Nishimura, and K. Mima, Plasma Phys. Controlled Fusion 41, A75 (1999).

${ }^{5}$ H. F. Robey, J. O. Kane, B. A. Remington, R. P. Drake, O. A. Hurricane, H. Louis, R. J. Wallace, J. Knauer, P. Keiter, and D. Arnett, Phys. Plasmas 8, 2446 (2001).

${ }^{6} \mathrm{P}$. Baclet, A. Benuzzi-Mounaix, S. Bouquet et al., Proceedings of the International Conference on Fusion Science and Applications 99, edited by C. Labaune, W. J. Hogan, and K. A. Tanaka (Elsevier, New York, 1999), p. 231.

${ }^{7}$ S. V. Lebedev (private communication, March 2000).

${ }^{8} \mathrm{~J}$. W. Stewart, in McGraw-Hill Encyclopedia of Science and Technology (McGraw-Hill, New York, 1987), Vol. 5, p. 281; J. Catchpole and G. D. Fulford, ibid., p. 284.

${ }^{9}$ E. Buckingham, Phys. Rev. 4, 345 (1914).

${ }^{10}$ L. I. Sedov, Similarity and Dimensional Methods in Mechanics (Academic, New York, 1959).

${ }^{11}$ J. Lacina, Plasma Phys. 13, 303 (1970).

${ }^{12}$ B. B. Kadomtsev, Sov. J. Plasma Phys. 1, 296 (1975).

${ }^{13}$ J. W. Connor and J. B. Taylor, Nucl. Fusion 17, 1047 (1977).

${ }^{14}$ D. D. Ryutov, R. P. Drake, J. Kane, E. Liang, B. A. Remington, and W. M. Wood-Vasey, Astrophys. J. 518, 821 (1999).

${ }^{15}$ D. D. Ryutov, R. P. Drake, and B. A. Remington, Astrophys. J., Suppl. 127, 465 (2000)

${ }^{16}$ R. P. Drake, Phys. Plasmas 7, 4690 (2000).

${ }^{17}$ Q. Zhiang and M. J. Graham, Phys. Rev. Lett. 79, 2674 (1997).

${ }^{18}$ W. D. Arnett, J. N. Bahcall, R. P. Kirschner, and S. E. Woosley, Annu. Rev. Astron. Astrophys. 27, 629 (1989).

${ }^{19}$ W. Hillebrandt and P. Hoflich, Rep. Prog. Phys. 52, 1421 (1993).

${ }^{20}$ E. Muller, B. Fryxell, and D. Arnett, Astron. Astrophys. 251, 505 (1991).

${ }^{21}$ K. Kifonidis, T. Plewa, H-Th. Janka, and E. Müller, Astrophys. J. Lett. 531, $123(2000)$

${ }^{22}$ J. Kane, D. Arnett, B. A. Remington, S. G. Glendinning, G. Bazan, R. P. Drake, B. A. Fryxell, R. Tessier, and K. Moore, Phys. Plasmas 6, 2065 (1999).

${ }^{23}$ G. Cecil, A. S. Wilson, and R. B. Tully, Astrophys. J. 390, 365 (1992).

${ }^{24}$ S. Heathcote, J. A. Morse, P. Hartigan, B. Reipurth, R. D. Schwartz, J. Bally, and J. M. Stone, Astron. J. 112, 1141 (1996).

${ }^{25}$ J. M. Blondin, Astrophys. J. 360, 370 (1990).

${ }^{26}$ J. M. Stone and M. L. Norman, Astrophys. J. 413, 198 (1993).

${ }^{27}$ L. M. Logory, P. L. Miller, and P. E. Stry, Astrophys. J., Suppl. 127, 423 (2000).

${ }^{28}$ D. R. Farley, K. G. Estabrook, S. G. Glendinning, S. H. Glenzer, B. A. Remington, K. Shigemori, J. M. Stone, R. J. Wallace, G. B. Zimmerman, and J. A. Harte, Phys. Rev. Lett. 83, 1982 (1999).

${ }^{29}$ K. Shigemori, R. Kodama, D. R. Farley, T. Koase, K. G. Estabrook, B. A. Remington, D. D. Ryutov, Y. Ochi, H. Azechi, J. Stone, and N. Turner, Astrophys. J. Lett. 533, 159 (2000).

${ }^{30}$ R. S. Sutherland and M. A. Dopita, Astrophys. J., Suppl. 88, 253 (1993).

${ }^{31}$ D. D. Ryutov, M. S. Derzon, and M. K. Matzen, Rev. Mod. Phys. 72, 167 (2000).

${ }^{32}$ S. Chandrasekhar, Hydrodynamic and Hydromagnetic Stability (Oxford University Press, Oxford, 1961). 
${ }^{33}$ K. O. Mikaelian, Phys. Rev. E 54, 3676 (1996).

${ }^{34}$ L. D. Landau and E. M. Lifshitz, Fluid Mechanics (Pergamon, New York, 1987).

${ }^{35}$ B. J. Cantwell, Annu. Rev. Fluid Mech. 13, 457 (1981).

${ }^{36}$ M. Van Dyke, An Album of Fluid Motion (Parabolic, Stanford, CA, 1982), p. 100.

${ }^{37}$ B. Gjevik, in Orographic Effects in Planetary Flows, Global Atmospheric Research Programme (GARP) Publication \#23, World Meteorological Organization-International Council of Scientific Unions (WMO/ICSU, 1980), p. 304.

${ }^{38}$ P. E. Dimotakis, J. Fluid Mech. 409, 69 (2000).

${ }^{39} \mathrm{~S}$. Woosley, in Supernovae, edited by A. G. Petschek (Springer-Verlag, New York, 1990), p. 182.

${ }^{40}$ M. Yamada, H. Ji, S. Hsu, R. Kulsrud, N. Bretz, F. Jobes, Y. Ono, and F. Perkins, Phys. Plasmas 4, 1936 (1997).

${ }^{41}$ L. Spitzer, Astrophys. J. 120, 1 (1954).

${ }^{42}$ E. A. Frieman, Astrophys. J. 120, 18 (1954).

${ }^{43}$ J. Hester, P. A. Scowen, R. Sankrit et al., Astron. J. 111, 2349 (1996).

${ }^{44}$ M. Pound, Astrophys. J. Lett. 493, 113 (1998).

${ }^{45}$ N. A. Levenson, J. R. Graham, I. S. McLean, E. E. Becklin, D. F. Figer,
A. M. Gilbert, J. E. Larkin, H. I. Teplitz, and M. K. Wilcox, Astrophys. J. Lett. 533, 53 (2000).

${ }^{46}$ D. D. Ryutov, K. S. Budil, S. G. Glendinning, B. A. Remington, R. P. Drake, and J. M. Stone, Bull. Am. Phys. Soc. 43, 1897 (1998).

${ }^{47}$ B. Lefloch and B. Lazareff, Astron. Astrophys. 289, 559 (1994).

${ }^{48}$ B. A. Remington, S. V. Weber, S. W. Haan, J. D. Kilkenny, S. G. Glendinning, R. J. Wallace, W. H. Goldstein, B. G. Wilson, and J. K. Nash, Phys. Fluids B 5, 2588 (1993).

${ }^{49}$ S. V. Weber, B. A. Remington, S. W. Haan, B. G. Wilson, and J. K. Nash, Phys. Plasmas 1, 3652 (1994).

${ }^{50}$ J. Lindl, Phys. Plasmas 2, 3933 (1995).

${ }^{51}$ D. D. Ryutov, submitted to Phys. Rev. Lett.

${ }^{52}$ L. O'C. Drury and J. T. Mendonca, Phys. Plasmas 7, 5148 (2000).

${ }^{53}$ D. E. Baldwin and D. D. Ryutov, Comments Plasma Phys. Control. Fusion 17, 1 (1995).

${ }^{54}$ E. P. Velikhov, I. V. Novobrantsev, V. D. Pis'mennyi, A. T. Rakhimov, and A. N. Starostin, Sov. Phys. Dokl. 17, 772 (1972).

${ }^{55}$ G. Hazak, A. L. Velikovich, M. Klapisch et al., Phys. Plasmas 6, 4015 (1999).

${ }^{56}$ H. J. Rood, Annu. Rev. Astron. Astrophys. 26, 245 (1988). 